\title{
Precise three-dimensional segmentation for breast region on magnetic resonance imaging
}

\author{
Tzu-Yu Liu ${ }^{1 *}$, Yu-Len Huang ${ }^{2}$, Dar-Ren Chen ${ }^{3}$ \\ 1, 2 Department of Computer Science, Tunghai University, Taichung, Taiwan \\ ${ }^{3}$ Comprehensive Breast Cancer Center, Changhua Christian Hospital, Changhua, Taiwan
}

\author{
Index Terms \\ Breast Cancer \\ Breast MRI \\ Image Segmentation \\ Horizontal and Vertical Projection
}

Received: 11 June 2016

Accepted: 12 July 2016

Published: 27 October 2016

\begin{abstract}
Contouring of breast region is an important step in computer aided analysis of Magnetic Resonance Imaging (MRI). The volume of breast region is helpful to physician in surgical operation. During the operation, physicians have to estimate the volume of the breast they removed by experience, then put the same weight of normal saline bag or silica gel in human body. Automatic contouring for breast region may assist physicians in making correct decision at surgical operation. However, automatic segmentation is difficult due to several resembling tissues around the breast region in MRI images. As breast MRI becomes more widespread, a functional automatic contouring method is essential and its clinical application is becoming urgent. For this purpose, this study presents a precise method to segment the breast region on each image slice. Then the obtained contour is used to estimate the volume of the breast. The pre-processing of proposed method reduces plenty of noises but still preserves the shape of breast. By using horizontal and vertical projection to extract the region of interest (ROI), the proposed method could obtain a precise contour of breast region.
\end{abstract}

(C) 2017 The Author(s). Published by TAF Publishing.

\section{INTRODUCTION}

Breast cancer is the most common cancer to women in the world. In 2015, about 40290 women and 440 men probably died from breast cancer [1]. Normally, if we can detect the cancer early, the cure rate would be raised [2]. It is important for early diagnosis that can decrease the mortality effectively.

With the advance of science and technology, Computer Aided Diagnosis (CAD) system is more widespread in current society. Radiologists also could acquire information that is helpful to them by CAD systems, such as the position of the breast or the volume of the breast, even the tumor information, etc. In the past times, physicians estimated the volume of the breast by their experience, but it is difficult for physicians with less experience of performing operations. With CAD system, even though physicians and radiologists are inexperienced, they would make a correct decision immediately.
In computer vision, image segmentation is the process to partition a digital image into several segments. The aim of image segmentation is to assist people in acquiring information from images. Image segmentation also is a typical method to locate objects or boundaries in images. In order to improve the of segmentation, image pre-processing plays an important role in whole algorithm. This study performed the pre-processing procedure which utilized the thresholding method, anisotropic filter [3] and morphological close operation to decrease the noise in breast MRI. After this step, the projection method was used to locate the rough pectoral and breast position. At last we computed the pixels of each slice of breast. Further, the volume of the breast would be obtained by accumulating pixels in all slices.

This study presented an automated algorithm based on projection to locate the breast to segment the desired ROI. By using projection techniques, the proposed method

\footnotetext{
${ }^{*}$ Corresponding author: Tzu-Yu Liu

†Email: g03350005@thu.edu.tw
} 
detected the contour of breast of each slice promptly. By grouping all the slices, the volume of the breast would be estimated. In the next section, the related works are introduced. In the third section, the proposed method and the materials will be described. Section four shows the result of this study. Finally, the conclusion of this study is presented in section five.

\section{RELATED WORK}

Segmentation methods of breast region could be roughly classified in certain groups: clustering-based, region-based, edge-based, the thresholding method, and the hybrid method with over two approaches. Clusteringbased method groups the pixels that have the same attributes such as K-means clustering method [4]. Regionbased approaches divide the image into split parts and connect the homogeneous regions for instance growing region. Edge-based methods used the edge detectors, such as [5] and Sobel (Works) to border the Region Of Interest (ROI). Thresholding method relied on the change of the grayscale for example the Otsu's method [6]. This study utilized the region-based method to detect the breast region. To define the region of the breast, projection techniques were performed to find the start points and the end points in the image. The task of breast segmentation in this study included two step: segment the upper part of image of the rough pectoral muscle and remove the air area. Wang et al. presented a region-based algorithm to detect the pectoral and breast boundary to extract the breast region [7]. Used K-means++ method to remove the background pixels [8]. Introduced a projection method to locate the position of breast area [9]. Utilized the edge-enhancement algorithm and the shortest path algorithm to determine the breast region [10].

\section{METHODOLOGY}

ROI generation is an absolutely necessary step for automatic segmentation in many CAD systems. Most of ROIs are usually irregular in shape which provide the rough location of breast area and exclude other tissues as much as possible. In order to get the Volume of Interest (VOI) of breast, the proposed method detected the ROI and calculated the number of pixels from each image slice. By accumulating the number of pixels per slice, the whole breast volume would be estimated. During detection of ROI in breast MR images, the regions that are insignificant such as lungs, heart and thoracic cavity must be eliminated cautiously to cut down the computation and promote the ac- curacy of segmentation. Therefore, a pre-processing procedure is essential in this study. After the pre-processing procedure, the proposed segmentation method utilized projection techniques to contour the breast region. Not only for the pectoral extraction but also for breast segmentation, projection techniques can locate the target accurately and rapidly.

\section{A. Data Acquisition}

Breast MRI was performed with the patient in the prone position. Examinations were performed with a 3.0-T commercially available system (Verio $®$; Siemens AG, Erlangen, Germany) and use of a dedicated 16-Channel breast coil. Imaging sequences included a localizing sequence, an axial tse_T1 weighted ( $3 \mathrm{~mm}$ ), tse_T2_tirm, pre, during, and post-Gd 3D-FSPGR $(1 \mathrm{~mm})$ with fat saturation images, before and five times after rapid bolus injection of $0.1 \mathrm{mmol} / \mathrm{L}$ gadobenate dimeglumine (Multihence $®$; Bracco, s.p.a., Milano, Italy) per kilogram of body weight at a rate of $2 \mathrm{ml} / \mathrm{s}$; followed by a saline flush, acquired at 60 -second interval. All obtained images were stored on the hard disk and transferred to a personal computer using a DICOM (Digital Imaging and Communications in Medicine) connection for image analysis.

\section{B. Image Pre-processing}

The outstanding pre-processing method makes the segmentation go smooth and retains the useful information. In this study, the thresholding method, anisotropic filter and morphological processing were performed as the pre-processing operations. The first step of the proposed pre-processing method, a thresholding method, assisted us to convert the grayscale images into the binary images that could get rid of the air area in image. By our experience, the gray-level value of the thresholding method would be 0.2 . The value is applicable to the subsequent step. The second step utilized the anisotropic filter and the morphology processing to treat with the delicate speckles that might influence the result of segmentation. Anisotropic filter could remove great obstacles and enhance the quality of images, and it could also preserve the boundary information that is important in segmentation phase. Moreover, morphology processing was applied to adjust the specifics of the image that was operated by anisotropic filter. The closing operation could smoothen the edge and fill the hole which was inside the breast area. The operation provided a positive effect for the proposed segmentation method. Figure 1 shows 
the original image and the pre-processed result. Figure 1(a) is an original MR image which contains abundant information and noises, such as the air area, the breast region, the

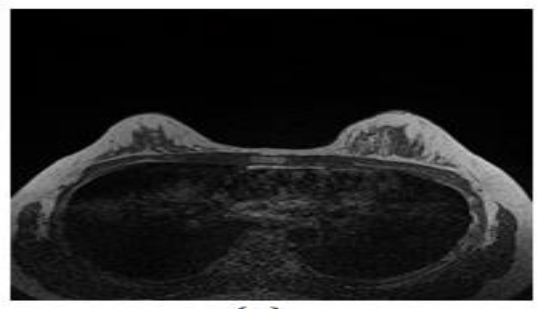

(a)

Fig. 1. (a) The original image, (b) The pre-processed image

\section{Horizontal Projection Segmentation}

The identification of the pectoral position is essential, because the organs under the pectoral are irrelevant to segment the breast region. Reducing the unnecessary area and making the segmentation go smooth are the purposes for the first step of segmentation. In order to achieve the target, the horizontal projection was performed in this study. The horizontal projection method obtains the histogram that is counted by the pixels whose value equals to one in the breast MRI. By this measure, the maximum value could be observed. Thus, the maximum value was set as the point of the demarcation. By the demarcating point, the image was separated into the upper region and the lower region.

\section{Vertical Projection Segmentation}

The vertical projection was made use of for separating the breast area and the tissues near the breast. Similar to the horizontal projection, the vertical projection method

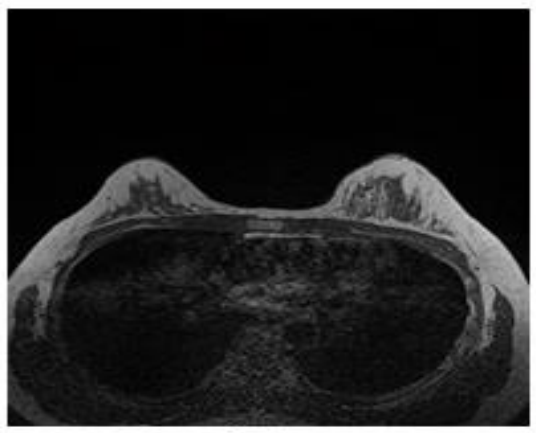

(a) organs in the body, etc. Figure 1(b) shows that the noises were removed in the image.

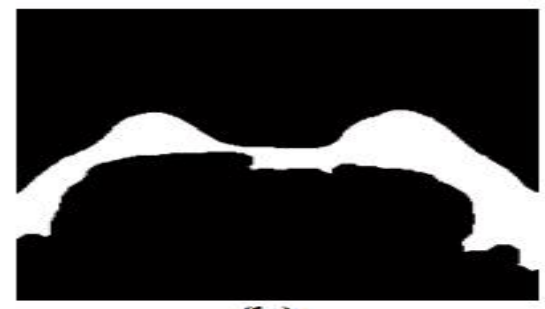

(b) was used to obtain the histogram that is counted by the white pixels in the breast MRI. Furthermore, the breast region could be segmented more precisely.

The vertical projection is utilized to confirm the range of breast, the histogram curve is similar to that of the breast image after pre-processing procedure. Therefore, the definition of the start point and the end point is based on the slope changes. If the slope is gradually increasing, the first point would be recorded as the start point. On the other hand, if the slope is gradually decreasing, the first point would be recorded as the end point.

Figure 2(a) displays the segmentation after the vertical projection. The red line is the start point of the breast, the blue line is the end point of the breast and the green line is the border between breast and the internal organs. Obviously, the rough breast boundary was detected. When the horizontal and vertical projections were completed, the breast region could be located. Figure 2(b) shows the preliminary result of projection segmentation.

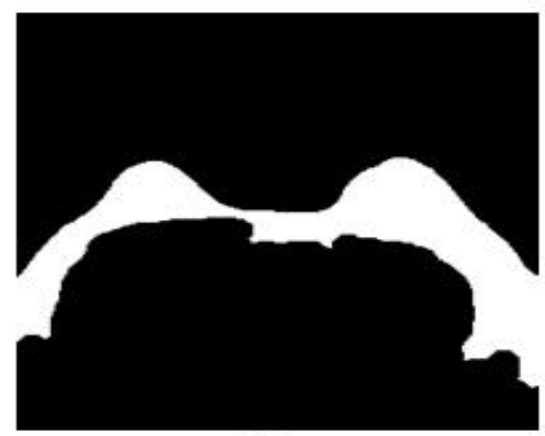

(b)

Fig. 2. Vertical projection breast segmentation. (a) Segmented breast area, (b) The breast region after horizontal and vertical projection segmentation 


\section{E. 3.5 Contouring Breast Region}

The preliminary breast region can be found by using the projection method. To optimize the accuracy, the region which is unconcerned with the breast area is supposed to remove. Next step, based on vertical projection segmentation, it is obvious to discriminate breast region from other tissue areas. In general, breast region contains parts of chest tissue which are not relative when physicians

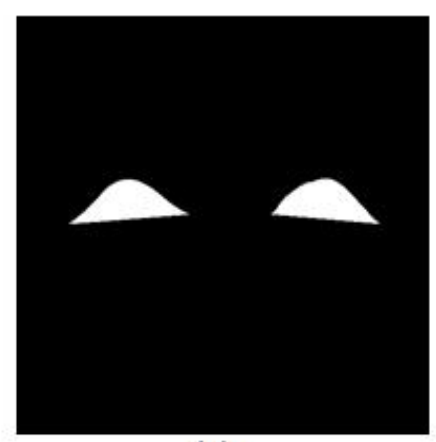

(a) operate the breast augmentation surgery. Due to the highest point in the inner breast and the lowest point in the outer breast, the lower bound was defined by these two points. The region above the lower bound is the precise breast region. Figure 3(a) shows the precise breast region. Figure 3(b) shows the all steps required to generate the result of the automatic segmentation in the original MRI.

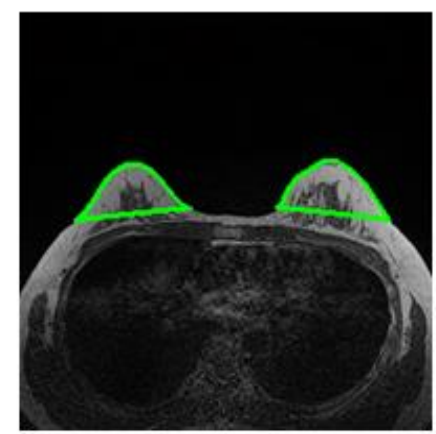

(b)

Fig. 3. The result of the automatic segmentation. (a) Precise breast region, (b) The result of the automatic segmentation in the original MR image
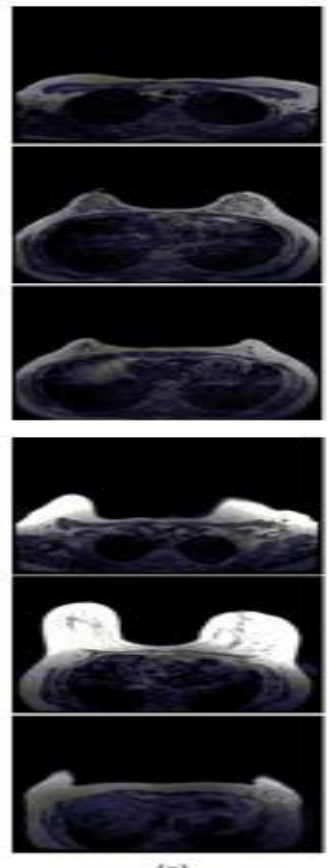

4a)
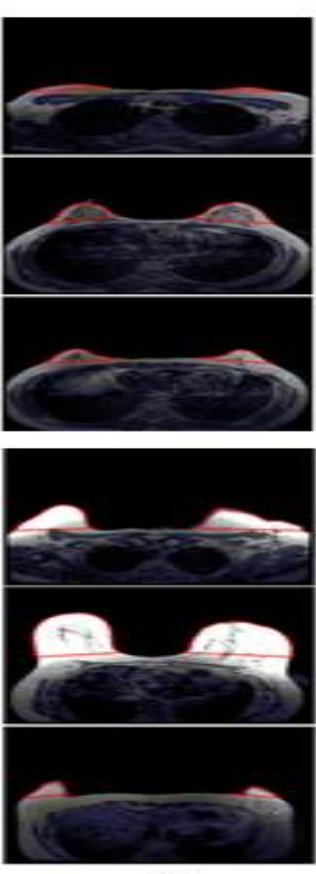

$\operatorname{los}$
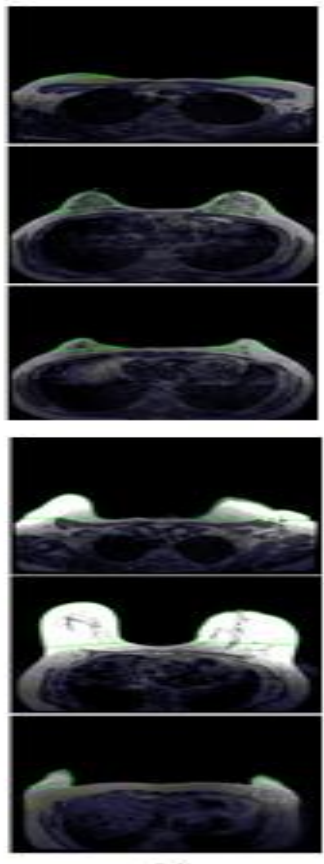

《<l

Fig. 4 . The result of proposed method. (a) The original DICOM images, (b) segmented result, (c) manually sketched 


\section{RESULTS}

\section{A. Dataset}

In the aggregate, 12 sets of MR breast images are used. The resolution of the nine sets is $512 \times 512 \times 60$ with 16 bits per pixel, the resolution of other two sets is $512 \times$ $512 \times 45$ with 16 bits per pixel. The resolution of the last one set is $448 \times 448 \times 60$ with 16 bits per pixel. Field of view (FOV) of the images is $320 \mathrm{~mm}$ and the slice thickness is 3 $\mathrm{mm}$. The proposed automatic segmentation method was implemented by Matlab (R2012a, MathWorks Inc., MA). The simulations were performed on a Windows PC with i7 (3.6 GHz) processor and 8GB memory. The boundary of the breast was manually sketched by experienced radiologists. The performance of the proposed algorithm was proved by comparing with the result of the radiologists' breast contour.

\section{B. Evaluation of Contour}

The four practical similarity measures, the Similarity Index (SI), Overlap Fraction (OF), Over Value (OV), and Extra Fraction (EF), between the manually sketched contours and the automatically determined contours are calculated for the quantitative analysis [1]. REF stands for the results delineated by the physician manually, and SEG represents the region of breast depicted by our automatic segmentation method. SI is the similar degree between REF and SEG. The overlap area comprises of the area of the proposed method and manual sketching by physician, extra area means the false positive area and missing area means false negative area. When SI, OF and OV are close to 1, and EF is close to 0 , it indicates that the breast area segmented by our method is similar to the result that is sketched by physician.

\section{Segmentation Results}

The segmentation results of proposed method are shown in Figure 4. Figure 4(a) shows the original breast MRI. Figure 4(b) shows the contours determined by our system. Figure 4(c) shows the region which is sketched manually by physician. Clearly, the breast region determined by the proposed system is similar to the sketching by physician indeed. Performance analysis was evaluated through the four practical similarity measures.

Table 1 shows the value of SI, OF, OV and EF for each case. The highest value of (SI, OF, OV) could reach $(0.95,0.93,0.93)$, and the lowest value of $(E F)$ is $(0.08)$ that means the segmentation result was similar to the delineation sketched by physician.

TABLE 1

The four similarity measures of all cases.

\begin{tabular}{lllll}
\hline \hline Case no. & SI & OF & OV & EF \\
\hline 1 & 0.88 & 0.83 & 0.80 & 0.20 \\
2 & 0.89 & 0.84 & 0.82 & 0.18 \\
3 & 0.94 & 0.93 & 0.90 & 0.10 \\
4 & 0.92 & 0.89 & 0.86 & 0.15 \\
5 & 0.91 & 0.86 & 0.85 & 0.15 \\
6 & 0.92 & 0.88 & 0.86 & 0.13 \\
7 & 0.93 & 0.91 & 0.87 & 0.14 \\
8 & 0.95 & 0.91 & 0.93 & 0.08 \\
9 & 0.91 & 0.88 & 0.85 & 0.16 \\
10 & 0.94 & 0.90 & 0.93 & 0.10 \\
11 & 0.94 & 0.90 & 0.89 & 0.10 \\
12 & 0.93 & 0.90 & 0.87 & 0.13 \\
\hline Average & 0.92 & 0.89 & 0.87 & 0.13 \\
\hline \hline
\end{tabular}




\section{DISCUSSION AND CONCLUSION}

This study demonstrated an efficient and rapid method to detect contours of breast region in MRI. This work could reduce the wrong decision by the radiologist who is inexperienced. The proposed method used thresholding method, anisotropic filter and morphological processing as the pre-processing procedure to decrease the noises and retain the shape of the breast. The segmentation method based on the projection techniques was performed to distinguish breast region from other tissues. The contours determined by the proposed method achieved the high similarity to manual sketched contours. The proposed segmentation method is valuable for physicians to make decision during the operation.

The performance analysis was examined by practical similarity measures in Table 1 . The average SI reaches 0.92 and the average $\mathrm{EF}$ is 0.13 . It means that the segmentation method proposed by our study is outstanding and trustworthy. It shows high potential to be necessary in computeraided analysis systems.

Besides, the segmentation result was confirmed by physicians that is valuable for not only the breast augmentation surgery but the breast cancer research. However the improvements are still required. The performance of a few cases was unsatisfied. In near future, the proposed method would be finely adjusted to assure the accuracy of the horizontal and vertical projection segmentation to avoid the boundary of the breast being in correct position.

\section{ACKNOWLEDGMENTS}

The authors would like to thank the Ministry of Science and Technology of the Republic of China (Taiwan) for financially supporting this research under Contract No. MOST 104-2221-E-029-016.

\section{REFERENCES}

[1] American Cancer Society, "Breast cancer facts and figures 2015-2016," 2016. [Online]. Available: goo.gl/0s7thz

[2] T. Berber, A. Alpkocak, P. Balci and O. Dicle, "Breast mass contour segmentation algorithm in digital mammograms," Computer Methods and Programs in Biomedicine, vol. 110, no. 2, pp. 150-159, 2013. DOI: 10.1007/s10798-013-9255-7.

[3] P. Perona and J. Malik, "Scale-space and edge detection using anisotropic diffusion," Pattern Analysis and Machine Intelligence, IEEE Transactions on, vol. 12, no. 7, pp. 629-639, 1990. DOI: 10.1109/34.56205.

[4] L. Wang and C Pan, "Robust level set image segmentation via a local correntropy-based K-means clustering," Pattern Recognition, vol. 47, no. 5, pp. 1917-1925, 2014. D0I: 10.1016/j.patcog.2013.11.014.

[5] J. Canny, "A computational approach to edge detection," IEEE Transactions on Pattern Analysis and Machine Intelligence, vol. 8, no. 6, pp. 679-698, 1986. DOI: 10.1109/TPAMI.1986.4767851.

[6] N. Otsu, "Threshold selection method from gray-level histograms," IEEE Transactions on Systems Man and Cybernetics, vol. 9, no. 1, pp. 62-66, 1979. DOI: 10.1109/TSMC.1979.4310076.

[7] L. Wang, B. Platel, T. Ivanovskaya, M. Harz and H. K. Hahn, "Fully automatic breast segmentation in 3D breast MRI," 9th IEEE International Symposium on, Biomedical Imaging (ISBI), 2012. DOI: 10.1109/isbi.2012.6235732.

[8] J. A. Rosado-Toro, T. Barr, J. P. Galons, M. T. Marron, A. Stopeck, C. Thomson, C., . . . J. J. Rodriguez, " Automated segmentation of breast fat-water MR images using empirical analysis," in IEEE International Conference on Acoustics, Speech and Signal Processing (ICASSP), 2013.

[9] S. K. Swee, C. F. Keong, C. S. Siang, T. C. Peng, S. F. Abbas and S. Omar, "Projection based region of interest segmentation in breast MRI images," International Journal on Advanced Science, Engineering and Information Technology, vol. 1, no. 1, pp. 113-116, 2011. DOI: 10.18517/ijaseit.1.1.26.

[10] J. Milenković, O. Chambers, M. M. Mušič and J. F. Tasič, "A utomated breast-region segmentation in the axial breast MR images," Computers in Biology and Medicine, vol. 62, pp. 55-64, 2015. DOI: 10.1016/j.compbiomed.2015.04.001.

[11] P. Anbeek, K. L. Vincken, M. J. van Osch, R. H. Bisschops and J. van der Grond, "Probabilistic segmentation of white matter lesions in MR imaging," Neuro Image, vol. 21, no. 3, pp. 1037-1044, 2004.

DOI: 10.1016/j.neuroimage.2003.10.012. 